\title{
CDA-OPTIMUM DESIGN FOR PARAMETER ESTIMATION, MINIMIZING THE AVERAGE VARIANCE AND ESTIMATING THE AREA UNDER THE CURVE
}

\author{
Mohamed M.E. Abd El-Monsef\&Sally M. El-Sobky \\ Faculty of Science, Tanta University \\ mmezzat@science.tanta.edu.eg \\ Faculty of Science, Tanta University \\ sally.mohamed@science.tanta.edu.eg
}

ABSTRACT

The aim of this paper is to introduce a new compound optimum design namedCDA, by combining the C-optimality, Doptimality, and A-optimality together. The significance of the proposed compound gains from that it can be used for parameter estimation, minimizing the average variance and model estimation simultaneously.

KEYWORDS: optimum design; C-optimality; D-optimality; A-optimality; compound criteria.

\section{Council for Innovative Research}

Peer Review Research Publishing System

Journal: JOURNAL OF ADVANCES IN MATHEMATICS

Vol .11, No.8

www.cirjam.com, editorjam@gmail.com 


\section{INTRODUCTION}

Cook and Wong [2] considered a compound optimality criterion that is a convex combination of the two concave criteria and so we can find the optimal design directly as if this is a single objective optimal design problem. D-optimality focuses on the variances of the estimates of the coefficients in the model, which minimizing the determinant of $\left(X^{T} X\right)^{-1}$ which is equivalent to maximizing thedeterminant of $X^{T} X$. An exact design is called D-optimal, if it minimizes the determinant $\mathrm{D}$ of the covariance matrix. C-optimality interest is in estimating the linear combination of the parameters $c^{T} \beta$ with minimum variance, where $\mathrm{c}$ is a known vector of constants. In A-optimality $\operatorname{tr} M^{-1}(\xi)$, the total variance of the parameter estimates, is minimized, equivalent to minimizing the average variance. This paper is organized as follows; the C -, D -, A - Optimum Designs were introduced in Section 2. The CDA-optimality was derived in section 3 and some of its properties were discussed. The generalized CDA- Optimum Design was introducedin Section 4.

\section{C -, D -, A - OPTIMUM DESIGNS}

C-optimality introduced by Elfving [2] which provided a geometrical interpretation for finding c-optimal designs and developed by Silvey and Titterington [10] and Titterington[11]. Fellman [4]justified that at most $m$ linearly independent support points are needed for a c-optimal design. Pukelsheim and Torsney [9]introduced a method for computing c-optimal weights given the support points. C-optimality minimize the variance of the best linear unbiased estimate for a given linear combination of the model parameters $c^{T} \theta$, where $c$ is $p \times 1$, a vector of a known constants. The c-optimaity criterion to be minimized is thus

$$
\operatorname{var} c^{T} \hat{\theta} \propto c^{T} M^{-1}(\xi) c
$$

The aim of c-optimality is to obtain the best design for estimating the linear combination of the parameters

$$
c_{1} \theta_{1}+\cdots+c_{p} \theta_{p}=c^{T} \theta
$$

The efficiency of any design $\xi$ relative to C-optimum design is defined as:

$$
\operatorname{Eff}^{c}(\xi)=\frac{c^{T} M^{-1}\left(\xi_{c}^{*}\right) c}{c^{T} M^{-1}(\xi) c}
$$

C-optimality is defined as min var $\left(C^{T} \theta\right)$, which is proportional to $C^{T} M^{-1}(\xi) C$. A disadvantage of c-optimum designs is that they are often singular.

\section{D- Optimum Design}

D-optimum design is one of the most commonly used design criteria for linear regression model that is also known as the Determinant criterion. This criterion introduced by Wald [12], and later was called D-optimality by Kiefer and Wolfowitz [5]. The D-Optimality is the most common criterion due to numerous applications found in the literature; see for example, Latif and Zafar Yab [6]and Poursina and Talebi [8]. D-optimality criterion is just to maximize the determinant of the Fisher information matrix, $\left|X^{T} X\right|$, this means that the optimal design matrix $X^{*}$ contains the $\mathrm{n}$ experiments which maximizes the determinant of $X^{T} X$.

Mathematically,

$$
\left|X^{* T} X^{*}\right|=\max \left(\left|X^{T} X\right|\right)
$$

Maximizing the determinant of the information matrix $X^{T} X$ is equivalent to minimizing the determinant of the dispersion matrix $\left(X^{T} X\right)^{-1}$. Using such an idea, the D-efficiency of an arbitrary design, $X$, is naturally defined as

$$
\operatorname{Eff}(D)=\left\{\frac{|M(\xi)|}{\mid M\left(\xi_{D}^{*}\right)}\right\}^{1 / P}
$$

\section{A-Optimum Design}

A-optimality criterion introduced by Chernoff [1]; who showed that the employed criterion of optimality is the one that involves the use of Fisher's information matrix. Invariance under re-parameterization loses its appeal if the parameters of interest have a definite physical meaning. Then the average-variance criterion provides a reasonable alternative. If the coefficient matrix is partitioned into its columns, $K=\left(c_{1}, \ldots, c_{s}\right)$, then the inverse $1 / \phi_{-1}$ can be represented as 
$\frac{1}{\phi_{-1}\left(C_{K}(A)\right)}=\frac{1}{s} \operatorname{tr}\left(C_{K}(A)^{-1}\right)=\frac{1}{s} \operatorname{tr}\left(K^{\prime} A^{-} K\right)=\frac{1}{s} \sum_{j \leq s} c_{j}^{\prime} A^{-} c_{i}$

This is the average of the standardized variances of the optimal estimatorsfor the scalar parameter systems $c_{1}^{\prime} \theta, \ldots, c_{s}^{\prime} \theta$ formed from the columns of $\mathrm{K}$. From the point of view of computational complexity, the criterion $\phi_{-1}$ is particularly simple to evaluate since it only requires the computation of the $s$ diagonal entries of the dispersion matrix $K^{\prime} A^{-} K$.

\section{CDA- OPTIMUM DESIGN}

To obtainparameter estimation, minimizing the average variance and model estimation of the area under the curve, a new compound criteria called $C D A$ is introduced.CDA is constructedby combing $\mathrm{C}, \mathrm{D}$ and A-optimality. By maximizing a weighted product of the efficiencies

$$
\left\{E f f^{(c)}\right\}^{k} \cdot\left\{E f f^{(D)}\right\}^{k(1-k)} \cdot\{E f f(A)\}^{(k-1)^{2}}
$$

Then taking the logarithm we get

$$
\begin{aligned}
& k \log \{E f f(c)\}+k(1-k)\{E f f(D)\}+(k-1)^{2}\{\text { Eff }(A)\} \\
&= k \log \left\{\frac{c^{T} M^{-1}\left(\xi_{c}^{*}\right) c}{c^{T} M^{-1}(\xi) c}\right\}+k(1-k) \log \left\{\frac{|M(\xi)|}{\mid M\left(\xi_{D}^{*}\right)}\right\}^{1 / P}+(k-1)^{2} \log \left\{\frac{\operatorname{tr} M^{-1}\left(\xi_{A}^{*}\right)}{\operatorname{tr} M^{-1}(\xi)}\right\} \\
&=-k \log \left\{c^{T} M^{-1}(\xi) c\right\}+k(1-k) \log \{M(\xi) \mid\}^{1 / P}+(k-1)^{2} \log \left\{\operatorname{tr} M^{-1}(\xi)\right\}
\end{aligned}
$$

The terms containing $\xi_{c}^{*}, \xi_{D}^{*}$ and $\xi_{A}^{*}$ are constants, a maximum is found over $\xi$. Hence, thecriterion that has to be maximized is given by

$$
\Phi^{(C D A)}(\xi)=-k \log \left\{c^{T} M^{-1}(\xi) c\right\}+\frac{k(1-k)}{p} \log \left\{M^{-1}(\xi)\right\}+(k-1)^{2} \log \left\{\operatorname{tr} M^{-1}(\xi)\right\}
$$

and the derivative function for CDA-optimality is

$$
\phi^{(C D A)}(x, \xi)=\frac{-k\left\{f^{T}(x) M^{-1}(\xi) c\right\}^{2}}{c^{T} M^{-1}(\xi) c}+\frac{(k-1)^{2}}{p}\left\{f^{T}(x) M^{-1} f(x)\right\}+(k-1)^{2}\left\{f^{T}(x) M^{-2} f(x)\right\}
$$

A CDA-optimum design, $\xi_{C D A}^{*}$, maximizes $\Phi_{C D A}(\xi)$ or equivalently $\log \Phi_{C D A}(\xi)$. The equivalence theorem can now be stated as follows:

\section{Theorem 1.}

i. A necessary and sufficient condition for a design $\xi_{C D A}^{*}$ to be CDA-optimum is fulfillment of the inequality $\phi^{(C D A)}\left(x, \xi_{C D A}^{*}\right) \leq 1, x \in \chi$.

ii. The upper bound of $\phi^{(C D A)}\left(x, \xi_{C D A}^{*}\right)$ is achieved at the points of the optimum design.

iii. For any non-optimum design $\xi_{1}$ that is a design for which $\Phi^{(C D A)}(\xi)<\Phi^{(C D A)}\left(\xi_{C D A}^{*}\right)$ and $\sup _{x \in \chi} \phi^{(C D A)}\left(x, \xi_{C D A}^{*}\right)>1$.

A measure of efficiency of a design $\xi$ relative to a CDA-optimum design is given by

$$
\operatorname{Eff}_{C D K L}(\xi)=\frac{\Phi_{C D A}(\xi)}{\Phi_{C D A}\left(\xi_{C D A}^{*}\right)}
$$

The proof can be made directly, since $\Phi^{(C D A)}(\xi), 0 \leq k \leq 1$ is a convex combination of three optimum design criteria, so the CDA-criterion is also convex and satisfying convexity conditions. 


\section{Properties of CDA-Optimality}

A good design should give a small variance matrix, therefore the function $\Phi$ is related to the variance matrix, and should have following properties:

i. Non-negativity: $\Phi_{C D A}(M) \geq 0$,

ii. Isotonicity: if $\left(M^{*}-M\right)$ is a positive semi-definite matrix, then $\Phi\left[M^{*}\right] \geq \Phi[M]$.

iii. Positive homogeneity: $\Phi[k M]=\mathrm{k} \Phi[M] ; k>0$,

iv. Superadditivity: $\Phi\left[M+M^{*}\right] \geq \Phi[M]+\Phi\left[M^{*}\right]$.

The previous properties are important to define with a proper scaling the relative efficiency of an experiment (or a design with the matrix $M$ ) with respect to another reference experiment with $M^{*}$. Pazman [7]discussed some other optimality properties for small samples.

\section{THE GENERALIZED CDA-OPTIMALITY:}

A generalized CDA-criterion will be introduced as:

$$
\begin{aligned}
\Phi^{(G C D A)}(\xi)= & -\sum_{j=1}^{m} a_{j} \log \left\{A_{j}^{T} c^{T} M^{-1}(\xi) c A_{j}\right\}+\sum_{i=1}^{n} \frac{s_{i}}{b_{i}} \log \left\{\left|A_{i}^{T} M^{-1}(\xi) A_{i}\right|\right\} \\
& +\sum_{l=1}^{k} c_{l} \log \operatorname{tr}\left\{A_{l}^{T} M^{-1}(\xi) A_{l}\right\}
\end{aligned}
$$

where, $a_{j}, s_{i}, b_{i}$ and $c_{l}$ are sets of non-negative coefficients reflecting the importance of the parts of the design criteria.

\section{REFERENCES}

[1] Chernoff, H. (1953). "Locally optimal designs for estimating parameters". Annals of Mathematical Statistics, 24, 586602.

[2] Cook, R. and Wong, W. (1994). "On the equivalence of constrained and compound optimal designs". Journal of the American Statistical Association, 89, 687-92.

[3] Elfving, G. (1952). "Optimum allocation in linear regression theory". Annals of Mathematical Statistics, 23, $255-262$.

[4] Fellman, J. (1974). "On the allocation of linear observations". Commentationes Physico-Mathematicae, Helsinki, 44, 27-78.

[5] Kiefer, J. and Wolfowitz, J. (1959). "Optimum designs in regression problems". Annals of Mathematical Statistics, 30, 271-294.

[6] Latif, S. and Zafar Yab, M. (2015). "D-optimal designs for beta regression models with single predictor". Journal of Statistical Computation and Simulation, 85(9), 1709-1724.

[7] Pazman, A. (2007). "Criteria for optimal design of small-sample experiments with correlated observations". Kybernetika, 43(4), $453-462$.

[8] Poursina, D. and Talebi, H. (2014). "Modified D-optimal design for logistic model". Journal of Statistical Computation and Simulation, 84(2), 428-437.

[9] Pukelsheim, F. and Torsney, B. (1991). "Optimal weights for experimental designs on linearly independent support points". Annals of Statistics, 19, 1614-1625.

[10] Silvey, S. and Titterington, D. (1973). "A geometric approach to optimal design theory".Biometrika, 60, 15-19.

[11] Titterington, D. (1975). "Optimal design: some geometrical aspects of D-optimality". Biometrika, 62, 313-320.

[12] Wald, A. (1943). "On the efficient design of statistical investigations". Annals of Mathematical Statistics, 14, 134-140. 